\title{
Mutation in the Hair Cell Specific Gene POU4F3 Is a Common Cause for Autosomal Dominant Nonsyndromic Hearing Loss in Chinese Hans
}

\author{
Longxia He, ${ }^{1,2,3}$ Xiuhong Pang, ${ }^{4}$ Penghui Chen, ${ }^{1,2,3}$ Hao Wu, ${ }^{1,2,3,5}$ and Tao Yang ${ }^{1,2,3}$ \\ ${ }^{1}$ Department of Otolaryngology-Head and Neck Surgery, Xinhua Hospital, Shanghai Jiaotong University School of Medicine, \\ Shanghai, China \\ ${ }^{2}$ Ear Institute, Shanghai Jiaotong University School of Medicine, Shanghai, China \\ ${ }^{3}$ Shanghai Key Laboratory of Translational Medicine on Ear and Nose Diseases, Shanghai, China \\ ${ }^{4}$ Department of Otorhinolaryngology-Head and Neck Surgery, Taizhou People's Hospital, Jiangsu Province, China \\ ${ }^{5}$ Department of Otorhinolaryngology-Head and Neck Surgery, Shanghai Ninth People's Hospital, \\ Shanghai Jiaotong University School of Medicine, Shanghai, China
}

Correspondence should be addressed to Hao Wu; wuhao622@sina.cn and Tao Yang; yangtfxl@sina.com

Received 8 September 2016; Revised 26 October 2016; Accepted 2 November 2016

Academic Editor: Renjie Chai

Copyright (C) 2016 Longxia He et al. This is an open access article distributed under the Creative Commons Attribution License, which permits unrestricted use, distribution, and reproduction in any medium, provided the original work is properly cited.

\begin{abstract}
Autosomal dominant nonsyndromic hearing loss (ADNSHL) is extremely heterogeneous. So far the genetic etiological contribution of the gene POU4F3 associated with ADNSHL has been rarely reported. In our previous study, a c.603_604delGG mutation in the hair cell specific gene POU4F3 has been identified as the pathogenic cause in one of the seven Chinese Han ADNSHL families. In the present study, we performed targeted next-generation sequencing of 144 known deafness genes in another nine Chinese Han ADNSHL families and identified two more novel mutations in POU4F3, p.Leu311Pro and c.120+1G $>$ C, as the pathogenic cause. Clinical characterization of the affected individuals in these three families showed that the three POU4F3 mutations may lead to progressive hearing loss with variable ages of onset and degrees of severity. Our results suggested that mutations in POU4F3 are a relatively common cause (3/16) for ADNSHL in Chinese Hans, which should be routinely screened in such cases during genetic testing.
\end{abstract}

\section{Introduction}

Hearing loss is one of the most common sensorineural defects, which may result from a great variety of genetic and environmental factors. Based on the inheritance patterns, the genetic hearing loss can be classified into autosomal recessive, autosomal dominant, and X-linked/mitochondrial, accounting for approximately $80 \%, 15 \%$, and less than $5 \%$ of nonsyndromic hearing loss, respectively [1]. Both autosomal dominant (ADNSHL) and autosomal recessive (ARNSHL) nonsyndromic hearing loss have an extremely high degree of heterogeneity. For the former, 35 causative genes and over 60 loci have been reported for ADNSHL (The hereditary Hearing Loss Homepage, http://hereditaryhearingloss.org/). So far, mutations in most ADNSHL genes were reported based on studies of the individual cases or families. The etiological contribution of the ADNSHL gene POU4F3 has been rarely studied. In recent years, however, the development of nextgeneration sequencing (NGS) has complemented the traditional Sanger sequencing method and made it possible to screen all deafness-associated genes in a high throughout manner [2-4].

Mutations in POU4F3 have been reported to lead to ADNSHL named as DFNA15 [5]. This gene encodes a 338 amino acids' POU family transcription factor with two conserved DNA-binding domains (the POU-specific domain and the POU homeodomain, amino acids $179-256$ and 274-333, resp.). In mouse inner ear, Pou $4 \mathrm{f} 3$ is strongly expressed in both inner and outer hair cells [6-9]. It activates the transcription of a downstream target gene Gfil, whose expression 
is required for the maintenance of the outer hair cells [10]. To date, only eleven different mutations in POU4F3 have been reported in Israeli Jewish, Dutch, Korean, Japanese, and Chinese ADNSHL families [5, 11-17]. The hearing loss caused by POU4F3 mutations was highly variable in the age of onset, the progression course, and the severity of hearing impairment.

In the previous $(n=7)$ and the present $(n=9)$ studies [18], we preformed targeted NGS of known deafness genes in 16 Chinese Han ADNSHL families and identified novel mutations in POU4F3 as the pathogenic cause in three of them. Characterization of the hearing phenotype was performed in the affected family members of these three families in the present study. Our results expanded the mutation spectrum of DFNA15 and suggested that mutations in POU4F3 are a relatively common cause for ADNSHL in Chinese Hans.

\section{Materials and Methods}

2.1. Subjects. Probands of sixteen Chinese Han families segregating ADNSHL were recruited through Xinhua Hospital, Shanghai, China. The pedigrees of the families were shown in Figure 1(a) and Supplementary Figure S1 in Supplementary Material available online at http://dx.doi.org/10.1155/ 2016/9890827. For Families P1748, PD6, and P59 in which mutations in POU4F3 were identified, 8, 10, and 13 additional family members were subsequently recruited, respectively. Informed consent was obtained from all subjects. This study was approved by the Ethics Committee of Xinhua Hospital, Shanghai Jiaotong University School of Medicine, Shanghai, China.

2.2. Clinical Evaluations. A detailed physical and medical history examination was performed in all probands of the ADNSHL families. The hearing levels were measured by pure tone audiometry (PTA) and shown as the average thresholds of $0.5,1,2$, and $4 \mathrm{kHz}$ from the better ear. The hearing levels were classified as normal $(<20 \mathrm{~dB})$, mild $(20-40 \mathrm{~dB})$, moderate (41-70 dB), severe (71-90 dB), and profound (>90 dB).

2.3. Mutation Analysis. Targeted NGS of 144 known deafness genes (see complete list in Supplementary Table S1) was performed using the MyGenotics gene enrichment system (MyGenotics, Boston, MD, USA) and the Illumina HiSeq 2000 sequencer (Illumina, San Diego, CA, USA) as previously described [18]. The raw data were first analyzed to filter out the low quality reads. NCBI37/hg19 assembly was used as the reference sequences. Nonsynonymous, on-target variants with maximum minor allele frequency (MAF) less than 0.001 in public databases NHLBI Exome Sequencing Project (ESP, http://evs.gs.washington.edu/EVS/) and the Exome Aggregation Consortium (ExAC, http://exac.broadinstitute.org/) were considered as the candidate pathogenic mutations in compliance with the ADNSHL inheritance. The pathogenicity of the candidate variants was predicted by computational programs Mutation Taster (http://www.mutationtaster.org/), PolyPhen-2 (http://genetics.bwh.harvard.edu/pph2/), PROVEAN, and SIFT (http://sift.jcvi.org/, cutoff scores set at -2.5 and 0.05 , resp.). Cosegregation of the pathogenic mutations and the hearing phenotype was verified in members of Families P1748 and PD6 by Sanger sequencing.

\section{Results}

3.1. POU4F3 Mutations Identified in the ADNSHL Families. In our previous studies of 7 Chinese Han ADNSHL families, a c.603_604delGG (p.Leu201fs*12) mutation in POU4F3 has been identified by targeted NGS as the pathogenic cause in Family P59 [18]. In the present study, we performed a comprehensive mutation screening in probands of another nine Chinese Han ADNSHL families (marked in asterisks in Figure 1(a) and Supplementary Figure S1) by targeted NGS of 144 known deafness genes. Interestingly, two novel heterozygous variants p.Leu311Pro (c.932T $>C$ ) and c.120+1G $>C$ in POU4F3 were identified as the candidate pathogenic variants in probands P1748-III-1 and PD6-IV-1, respectively (Table 1), along with a heterozygous TECTA p.Val1830Met and a heterozygous TMC1 p.Ser697X variant in proband P1748-1 (Table 1). Sanger sequencing in the rest of the family members confirmed that p.Leu311Pro and c. $120+1 \mathrm{G}>\mathrm{C}$ were the only two candidate variants segregating with the hearing phenotype in Families P1748 and PD6 (Figures 1(a) and 1(b)), while the TECTA p.Val1830Met and the TMC1 p.Ser697X variants were not seen in any other affected family members of P1748. The p.Leu311Pro (c.932T $>C$ ) and c.120+1G $>C$ in POU4F3 were not reported in previous studies, not present in the NHLBI $\operatorname{ESP}(n=6503)$ and $\operatorname{ExAC}(n=60706)$ database, and not seen in 300 Chinese Han normal hearing controls. The c. $120+1 \mathrm{G}>\mathrm{C}$ mutation was predicted to abolish the $5^{\prime}$ splice site of introns 1 of POU4F3 (Figure 2(a)). The p.Leu311Pro mutation substituted a conserved amino acid Leu311 (Figure 2(b)) and was predicted to be deleterious by computational programs Mutation Taster, PolyPhen-2, PROVEAN, and SIFT (Table 1).

3.2. Clinical Characteristics of the Three ADNSHL Families with POU4F3 Mutations. Based on the audiograms and the self-description of the affected individuals with c.603. 604delGG, p.Leu311Pro, and c.120+1G $>C$ mutations in $P O U 4 F 3$, the hearing loss associated with the POU4F3 mutations was typically progressive with considerable variability in ages of onset and degree of severity both interfamilially and intrafamilially. In Family P1748 with the p.Leu311Pro mutation, proband III-1 had notable hearing loss since age 10 years. The hearing loss affected high frequency most and gradually progressed to moderate hearing loss at age 29 years (Figure $1(\mathrm{c})$ ). The affected individuals in the second generation of Family P1748 had hearing loss at the onset age of 10 years to 20 years and all eventually progressed to profound after age 50 years. Interestingly, all six female patients in the second generation had significantly decreased hearing levels after giving birth to their children. In Family PD6 with the c.120+1G>C mutation, proband IV-1 had congenital, moderate hearing loss with a relatively "flat" audiometric profile at age 23 years affecting all frequencies (Figure 1(c)). Other affected individuals in this family demonstrated progressive, moderate-toprofound deafness depending on their ages. The ages of onset 

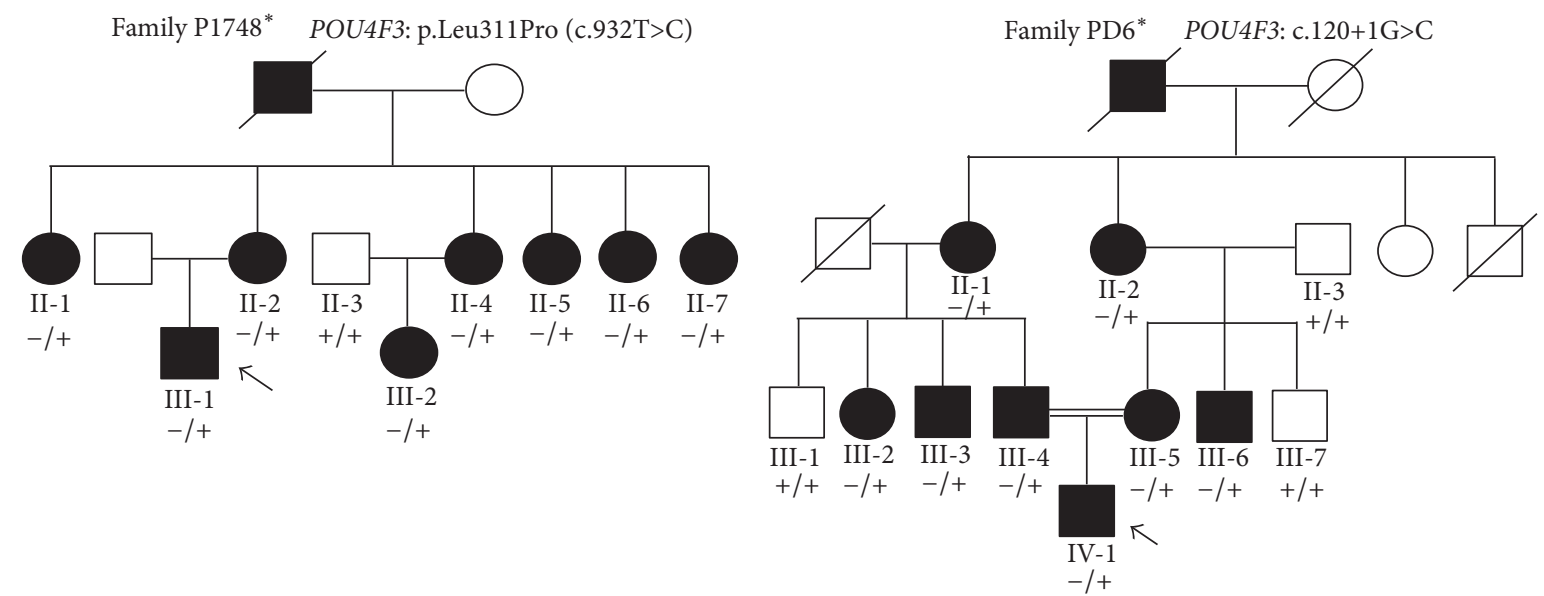

(a)

Family P1748 POU4F3: c.932T >C

T C G C T G A G AAAC T G G A C C T TAAAA A

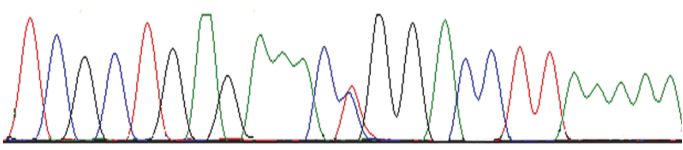

POU4F3: wild type

T C G C T GA GAAACT G GACCT TAAAAA

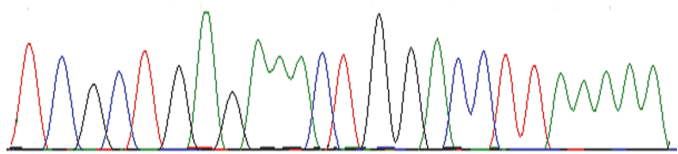

Family PD6 POU4F3: c. $120+1 \mathrm{G}>\mathrm{C}$

A G C C C C G C A G C T A C G T A G T G G

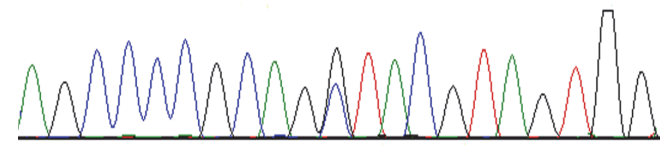

POU4F3: wild type

\begin{tabular}{lllllllllllllllllllll} 
A & $G$ & $C$ & $C$ & $C$ & $C$ & $G$ & $C$ & $A$ & $G$ & G & $T$ & $A$ & $C$ & $G$ & $T$ & $A$ & $G$ & $T$ & $G$ & $G$ \\
\hline
\end{tabular}

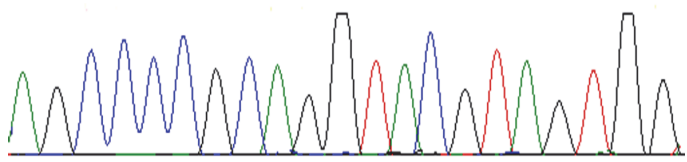

(b)

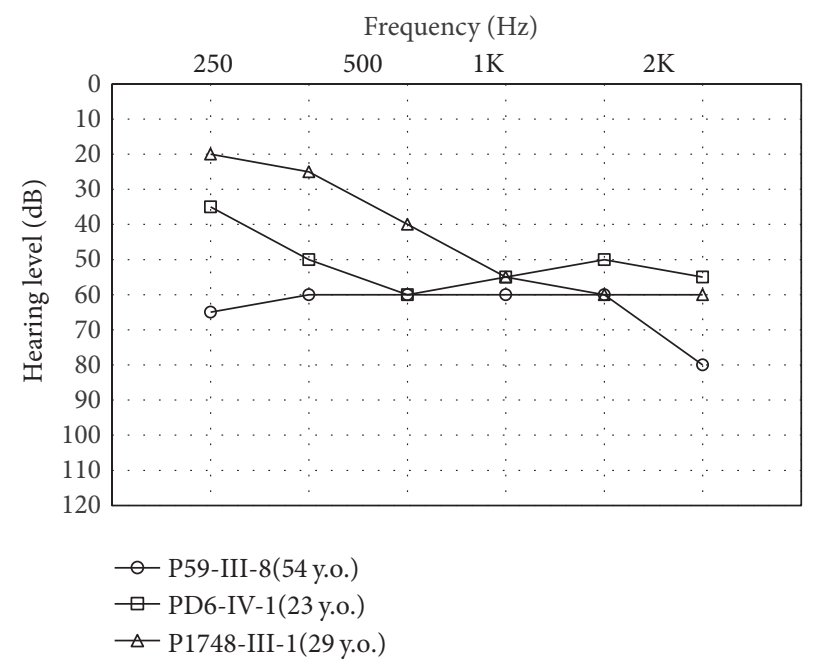

(c)

FIgure 1: POU4F3 mutations identified in the Chinese Han ADNSHL families. (a) Pedigrees and genotypes of the families with POU4F3 mutations. Probands were pointed by arrows. - and + indicate the mutant and wild type alleles, respectively. Asterisks indicate the families with POU4F3 mutations identified in the present study. (b) Chromatograms showing the c.932T $>$ C (p.Leu311Pro) and the c.120+1G $>$ C mutations in POU4F3. (c) Audiograms of the probands of the three families. 
TABLE 1: Candidate pathogenic mutations identified in probands of Families P1748 and PD6 by targeted NGS.

\begin{tabular}{|c|c|c|c|c|c|c|c|c|c|}
\hline Proband & $\begin{array}{l}\text { Gene (reference } \\
\text { sequence) }\end{array}$ & Mutation & $\begin{array}{c}\text { MAF } \\
(\text { ExAC) }\end{array}$ & $\begin{array}{c}\text { MAF } \\
\text { (NHLBI } \\
\text { ESP) }\end{array}$ & $\begin{array}{l}\text { Mutation } \\
\text { Taster }\end{array}$ & $\begin{array}{l}\text { PROVEAN } \\
\text { (score) }\end{array}$ & SIFT (score) & $\begin{array}{c}\text { PolyPhen-2 } \\
\text { (HumVar } \\
\text { score) }\end{array}$ & $\begin{array}{c}\text { Intrafamilial } \\
\text { phenotype } \\
\text { cosegregation }\end{array}$ \\
\hline \multirow{3}{*}{ P1748-III-1 } & $\begin{array}{c}\text { POU4F3 } \\
\left(\mathrm{NM} \_002700\right)\end{array}$ & $\begin{array}{l}\text { p.Leu311Pro } \\
(\text { c.932T>C) }\end{array}$ & 0 & 0 & $\begin{array}{l}\text { Disease } \\
\text { causing }\end{array}$ & $\begin{array}{c}\text { Deleterious } \\
(-3.63)\end{array}$ & $\begin{array}{c}\text { Damaging } \\
(0)\end{array}$ & $\begin{array}{c}\text { Probably } \\
\text { damaging (1) }\end{array}$ & Yes \\
\hline & $\begin{array}{c}\text { TECTA } \\
(\text { NM_005422) }\end{array}$ & $\begin{array}{l}\text { p.Val1830Met } \\
\text { (c. } 5488 \mathrm{G}>\mathrm{A})\end{array}$ & 0.0003871 & 0 & $\begin{array}{l}\text { Disease } \\
\text { causing }\end{array}$ & $\begin{array}{l}\text { Neutral } \\
(-0.83)\end{array}$ & $\begin{array}{l}\text { Damaging } \\
(0.008)\end{array}$ & $\begin{array}{c}\text { Probably } \\
\text { damaging } \\
(0.969)\end{array}$ & No \\
\hline & $\begin{array}{c}\text { TMC1 } \\
\text { (NM_138691) }\end{array}$ & $\begin{array}{c}\text { p.Ser697X } \\
(\text { c. } 2090 \mathrm{C}>\mathrm{G})\end{array}$ & 0 & 0 & $\begin{array}{l}\text { Disease } \\
\text { causing }\end{array}$ & $\begin{array}{l}\text { Deleterious } \\
(-10.26)\end{array}$ & - & - & No \\
\hline PD6-IV-1 & $\begin{array}{c}\text { POU4F3 } \\
(\text { NM_002700) }\end{array}$ & c. $120+1 G>C$ & 0 & 0 & $\begin{array}{l}\text { Disease } \\
\text { causing }\end{array}$ & - & - & - & Yes \\
\hline
\end{tabular}

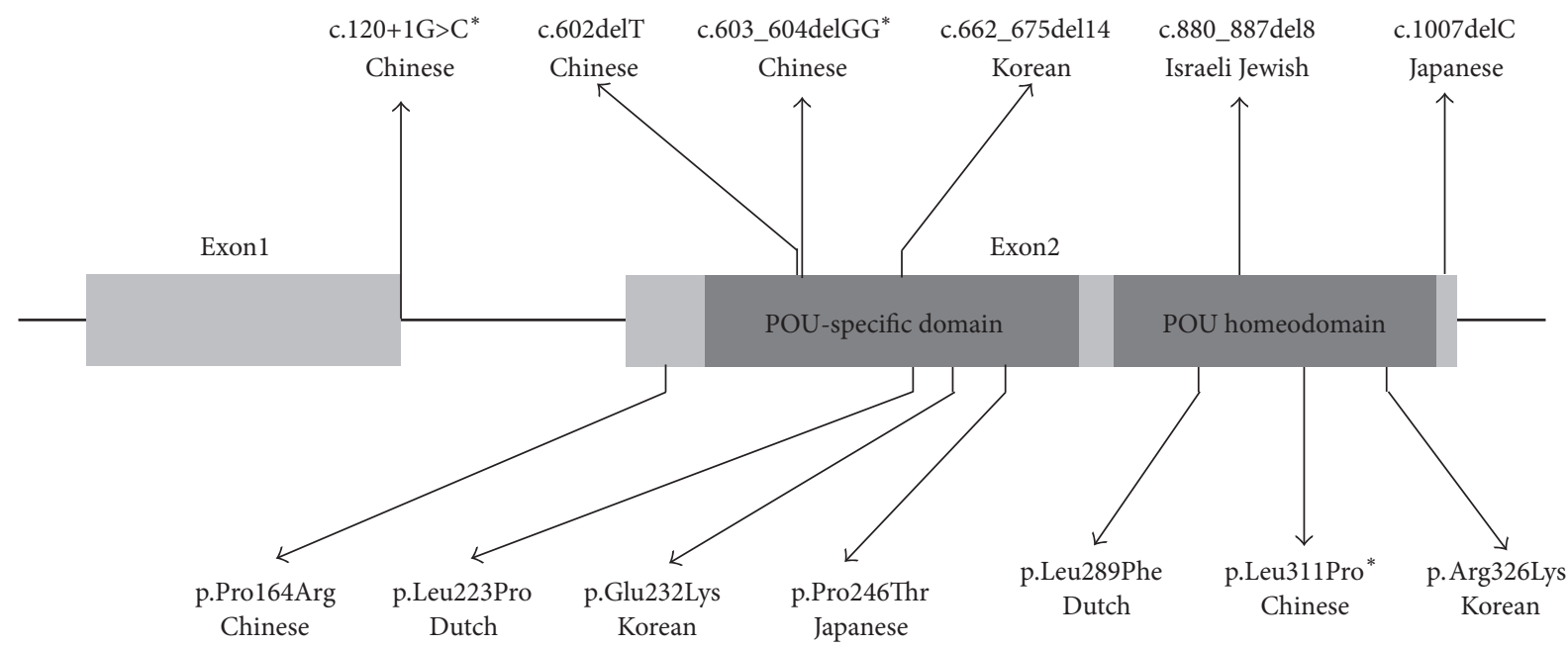

(a)

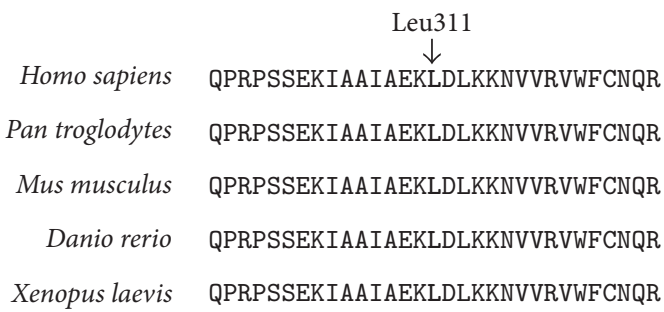

(b)

FIGURE 2: Summary and conservation of the POU4F3 mutations. (a) Schematic illustration of the thirteen reported POU4F3 mutations associated with DFNA15. Asterisks indicated the mutations reported in the present study. (b) Multispecies sequence alignment showing the evolutionary conserved amino acid Leu311.

ranged from congenital to 40 years. In Family P59 with the c.603_604delGG mutation, the proband III-8 had only moderate hearing loss at 54 years of age (Figure 1(c)) and all affected individuals in this family had a rather late age of onset around 40s. For other auditory symptoms, tinnitus has been complained by proband P1748-III-1. No vestibular dysfunction was shown in any affected individuals.

\section{Discussion}

Combined with our present and previous studies, we identified mutations in POU4F3 as the pathogenic cause of deafness in 3 of the 16 (18\%) Chinese Han ADNSHL families, suggesting that it is a relatively common cause for ADNSHL in Chinese Hans. Consistently, seven of the ten previously 
reported $P O U 4 F 3$ mutations from other research groups were also from the East Asians (three in Korean, two in Japanese, and two in Chinese, Figure 2(a)), suggesting that this gene should be routinely screened in ADNSHL cases of East Asian descent. In contrast, only three (one in Israeli Jewish and two in Dutch, Figure 2(a)) POU4F3 mutations were previously reported from regions other than East Asia. The distinguished mutation spectrum among different ethnical groups has also been reported for other deafness genes such as SLC26A4, in which case biallelic SLC26A4 mutations can be identified in $88.4 \%$ of deaf patients with nonsyndromic EVA in Chinese but only $15 \%$ in Caucasians $[19,20]$.

Our study also expanded the mutation spectrum of POU4F3. Figure 2(a) summarized the type, position, and associated ethnicity of the thirteen POU4F3 mutations reported to date. Four of them, including the c.603_604delGG (p.Leu201fs ${ }^{*} 12$ ) mutation reported in our previous study, were truncating mutations that were predicted to lead to prematurely stopped protein product or nonsense-mediated decay of the mRNA, while another seven POU4F3 mutations, including the p.Leu311Pro mutation reported in the present study, were missense mutations leading to single amino acid substitutions. Notably, these twelve mutations were all located within or close to the POU-specific domain or the POU homeodomain, the two conserved DNA-binding domains of POU4F3 encoded in exon 2. On the contrary, the c.120+1G>C mutation identified in the present study is the only reported mutation outside exon 2 of POU4F3.

Consistent with previous reports $[5,11,13-16]$, the POU4F3 mutations identified in the present study were associated with progressive hearing loss with considerable variability in the ages of onset and the degrees of severity, and this variable hearing phenotype can be seen both interfamilially and intrafamilially. Apparently no simple genotypephenotype correlation can be drawn based on the position or the truncating/nontruncating nature of the POU4F3 mutations. In a previous study of the Pou $4 \mathrm{f3}$ mutant deaf mice, deficiency of Pou4f3 has been found to result in reduced expression of its hair cell specific downstream target Gfil, which was suggested as the direct cause of the outer hair cell degeneration in the Pou $4 f 3$ mutant mice [10]. In future studies, therefore, it will be interesting to correlate the presumably reduced levels of the Gfi1 transcription with different POU4F3 mutations and the severity of the associated hearing loss.

\section{Conclusions}

Mutations in POU4F3 are a relatively common cause for ADNSHL in Chinese Hans. The hearing loss associated with POU4F3 mutations has considerable variability in the ages of onset and the degrees of severity.

\section{Competing Interests}

The authors declare no competing financial interests.

\section{Authors' Contributions}

Longxia He, Xiuhong Pang, and Penghui Chen contributed equally to this work.

\section{Acknowledgments}

This research was supported by grants from National Science Foundation of China (81570930 and 81371101 to Tao Yang; 81330023 to Hao Wu), Shanghai Municipal Science and Technology Commission (14DZ1940102 to Tao Yang; 14DZ2260300 and 14DJ1400201 to Hao Wu), and Shanghai Municipal Education Commission-Gaofeng Clinical Medicine Grant (20152519 to Tao Yang).

\section{References}

[1] R. J. H. Smith, A. E. Shearer, M. S. Hildebrand, and G. Van Camp, "Deafness and hereditary hearing loss overview," in GeneReviews $^{\circledR}$, R. A. Pagon, M. P. Adam, H. H. Ardinger et al., Eds., University of Washington, Seattle, Wash, USA, 1993.

[2] A. E. Shearer, A. P. DeLuca, M. S. Hildebrand et al., "Comprehensive genetic testing for hereditary hearing loss using massively parallel sequencing," Proceedings of the National Academy of Sciences of the United States of America, vol. 107, no. 49, pp. 21104-21109, 2010.

[3] A. E. Shearer and R. J. H. Smith, "Genetics: advances in genetic testing for deafness," Current Opinion in Pediatrics, vol. 24, no. 6, pp. 679-686, 2012.

[4] Z. Brownstein, Y. Bhonker, and K. B. Avraham, "High-throughput sequencing to decipher the genetic heterogeneity of deafness," Genome Biology, vol. 13, article 245, 2012.

[5] O. Vahava, R. Morell, E. D. Lynch et al., "Mutation in transcription factor $P O U 4 F 3$ associated with inherited progressive hearing loss in humans," Science, vol. 279, no. 5358, pp. 1950-1954, 1998.

[6] L. Erkman, R. J. McEvilly, L. Luo et al., "Role of transcription factors Brn-3.1 and Brn-3.2 in auditory and visual system development," Nature, vol. 381, no. 6583, pp. 603-606, 1996.

[7] S. W. Wang, X. Mu, W. J. Bowers et al., "Brn3b/Brn3c double knockout mice reveal an unsuspected role for Brn3c in retinal ganglion cell axon outgrowth," Development, vol. 129, no. 2, pp. $467-477,2002$

[8] M. Xiang, L. Gan, D. Li et al., "Essential role of POU-domain factor Brn-3c in auditory and vestibular hair cell development," Proceedings of the National Academy of Sciences of the United States of America, vol. 94, no. 17, pp. 9445-9450, 1997.

[9] M. Xiang, W.-Q. Gao, T. Hasson, and J. J. Shin, "Requirement for Brn-3c in maturation and survival, but not in fate determination of inner ear hair cells," Development, vol. 125, no. 20, pp. 39353946,1998

[10] R. Hertzano, M. Montcouquiol, S. Rashi-Elkeles et al., "Transcription profiling of inner ears from Pou $4 \mathrm{f}^{\text {ddl/ddl }}$ identifies Gfil as a target of the Pou4f3 deafness gene," Human Molecular Genetics, vol. 13, no. 18, pp. 2143-2153, 2004.

[11] R. W. J. Collin, R. Chellappa, R.-J. Pauw et al., "Missense mutations in POU4F3 cause autosomal dominant hearing impairment DFNA15 and affect subcellular localization and DNA binding," Human Mutation, vol. 29, no. 4, pp. 545-554, 2008. 
[12] H. Mutai, N. Suzuki, A. Shimizu et al., "Diverse spectrum of rare deafness genes underlies early-childhood hearing loss in Japanese patients: a cross-sectional, multi-center next-generation sequencing study," Orphanet Journal of Rare Diseases, vol. 8, article 172, 2013.

[13] H.-J. Kim, H.-H. Won, K.-J. Park et al., "SNP linkage analysis and whole exome sequencing identify a novel POU4F3 mutation in autosomal dominant late-onset nonsyndromic hearing loss (DFNA15)," PLoS ONE, vol. 8, no. 11, Article ID e79063, 2013.

[14] H. K. Lee, H. J. Park, K. Y. Lee, R. Park, and U. K. Kim, “A novel frameshift mutation of POU4F3 gene associated with autosomal dominant non-syndromic hearing loss," Biochemical and Biophysical Research Communications, vol. 396, no. 3, pp. 626-630, 2010.

[15] X. Z. Cai, Y. Li, L. Xia et al., "Exome sequencing identifies POU4F3 as the causative gene for a large Chinese family with non-syndromic hearing loss," Journal of Human Genetics, 2016.

[16] Q. Wei, H. Zhu, X. Qian et al., "Targeted genomic capture and massively parallel sequencing to identify novel variants causing Chinese hereditary hearing loss," Journal of Translational Medicine, vol. 12, article 311, 2014.

[17] M. Miyagawa, T. Naito, S.-Y. Nishio, N. Kamatani, and S.-I. Usami, "Targeted exon sequencing successfully discovers rare causative genes and clarifies the molecular epidemiology of Japanese deafness patients," PLOS ONE, vol. 8, no. 8, Article ID e71381, 2013.

[18] T. Yang, X. Wei, Y. Chai, L. Li, and H. Wu, "Genetic etiology study of the non-syndromic deafness in Chinese Hans by targeted next-generation sequencing," Orphanet Journal of Rare Diseases, vol. 8, no. 1, article 85, 2013.

[19] Q.-J. Wang, Y.-L. Zhao, A. Q. Rao et al., "A distinct spectrum of SLC26A4 mutations in patients with enlarged vestibular aqueduct in China," Clinical Genetics, vol. 72, no. 3, pp. 245-254, 2007.

[20] T. Yang, H. Vidarsson, S. Rodrigo-Blomqvist et al., "Transcriptional control of SLC26A4 is involved in Pendred syndrome and nonsyndromic enlargement of vestibular aqueduct (DFNB4)," American Journal of Human Genetics, vol. 80, no. 6, pp. 10551063, 2007. 

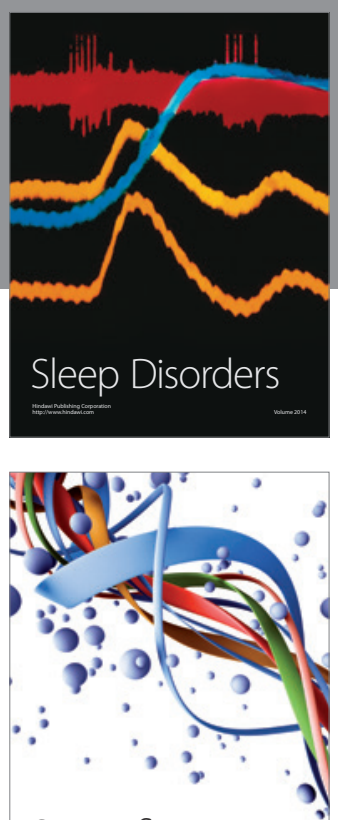

Scientifica
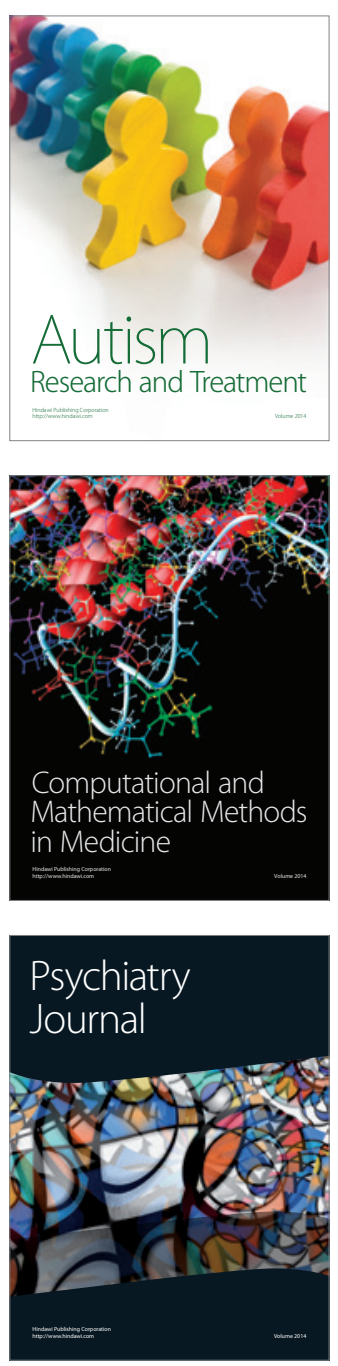
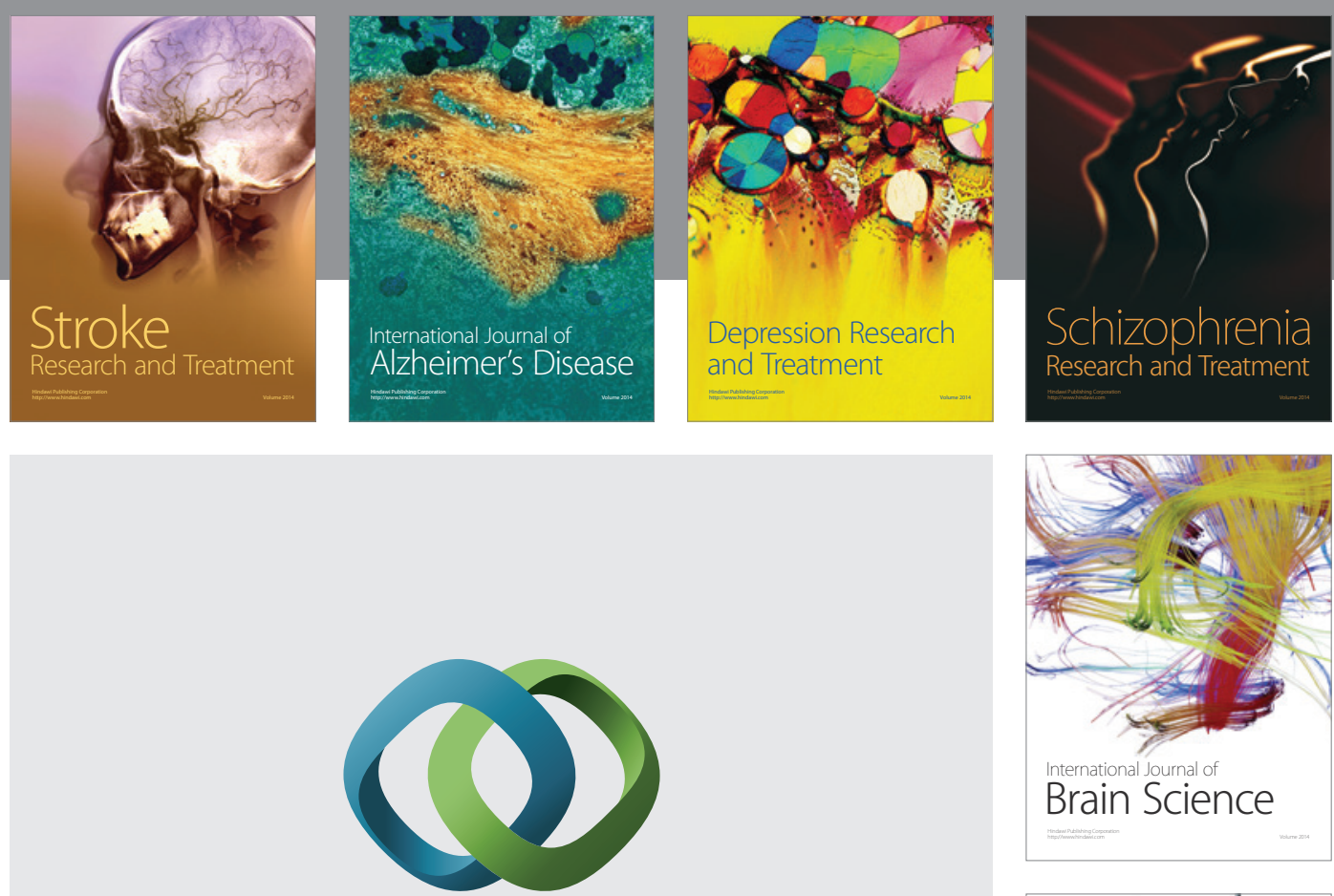

\section{Hindawi}

Submit your manuscripts at

http://www.hindawi.com
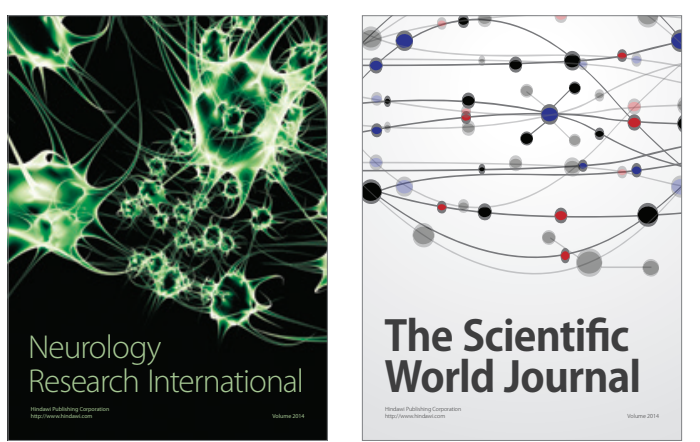

The Scientific World Journal

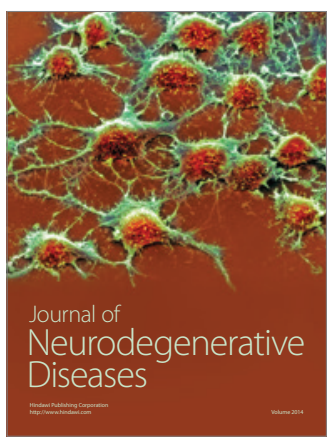

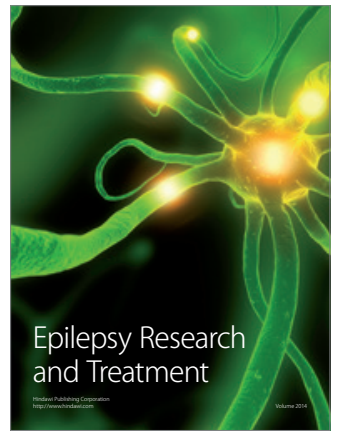

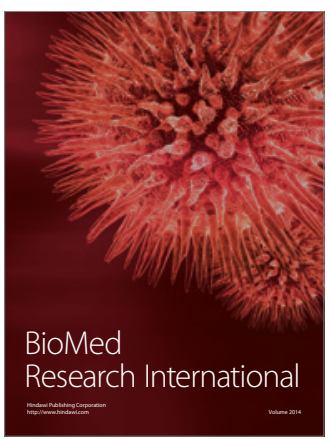

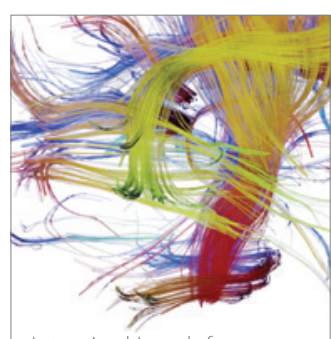

Brain Science

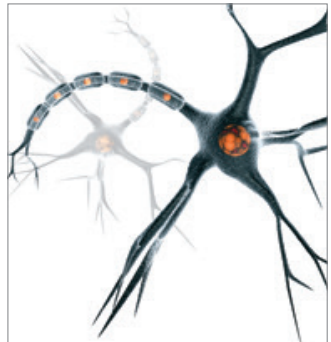

Neural Plasticity
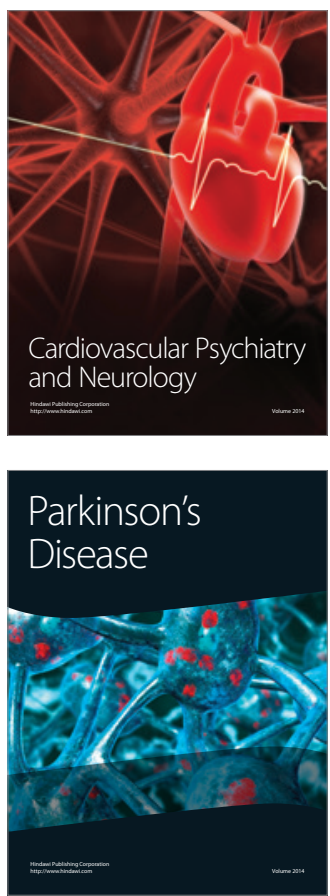